\title{
Safety of Esophageal Cancer Surgery During the First Wave of the COVID-19 Pandemic in Europe: A Multicenter Study
}

\author{
Alexander B. J. Borgstein, MD ${ }^{1}$, Stefanie Brunner, $\mathrm{MD}^{2}$, Masaru Hayami, MD, $\mathrm{PhD}^{3,4}$, Johnny Moons, $\mathrm{MSc}^{5}$, \\ Hans Fuchs, MD, $\mathbf{P h D}^{2}$, Wietse J. Eshuis, MD, $\mathbf{P h D}^{1}$, Suzanne S. Gisbertz, MD, $\mathbf{P h D}^{1}$, \\ Christiane J. Bruns, MD, $\mathrm{PhD}^{2}$, Philippe Nafteux, MD, $\mathrm{PhD}^{5}$, Magnus Nilsson, $\mathrm{MD}, \mathrm{PhD}^{3,4}$, \\ Wolfgang Schröder, MD, $\mathbf{P h D}^{2}$, and Mark I. van Berge Henegouwen, $\mathrm{MD}, \mathbf{P h D}^{1}$ \\ ${ }^{1}$ Department of Surgery, Amsterdam UMC, University of Amsterdam, Amsterdam, The Netherlands; ${ }^{2}$ Department of \\ General, Visceral, Cancer and Transplantation Surgery, University Hospital of Cologne, Cologne, Germany; ${ }^{3}$ Division of \\ Surgery, Department of Clinical Science, Intervention and Technology (CLINTEC), Karolinskja Institutet, Solna, Sweden; \\ ${ }^{4}$ Department of Upper Abdominal Diseases, Karolinska University Hospital, Stockholm, Sweden; ${ }^{5}$ Department of Surgery, \\ Universitair Ziekenhuis Leuven, Leuven, Belgium
}

\begin{abstract}
Background. Many hospitals postponed elective surgical care during the first wave of the coronavirus disease 2019 (COVID-19) pandemic. Some centers continued elective surgery, including esophageal cancer surgery, with the use of preoperative screening methods; however, there is no evidence supporting the safety of this strategy as postoperative outcomes after esophageal cancer surgery during the COVID-19 pandemic have not yet been investigated.

Methods. This multicenter study in four European tertiary esophageal cancer referral centers included consecutive adult patients undergoing elective esophageal cancer surgery from a prospectively maintained database in a COVID-19 pandemic cohort (1 March 2020-31 May 2020)
\end{abstract}

Alexander B. J. Borgstein and Stefanie Brunner shared first authorship and contributed equally to this article.

Wolfgang Schröder and Mark I. van Berge Henegouwen shared last authorship and contributed equally to this article.

Supplementary Information The online version contains supplementary material available at https://doi.org/10.1245/s10434021-09886-z.

(C) The Author(s) 2021

First Received: 14 December 2020

Accepted: 17 February 2021;

Published Online: 8 April 2021

M. I. van Berge Henegouwen, MD, PhD

e-mail: m.i.vanbergehenegouwen@amsterdamumc.nl and a control cohort (1 October 2019-29 February 2020). The primary outcome was the rate of respiratory failure requiring mechanical ventilation.

Results. The COVID-19 cohort consisted of 139 patients, versus 168 patients in the control cohort. There was no difference in the rate of respiratory failure requiring mechanical ventilation $(13.7 \%$ vs. $8.3 \%, p=0.127)$ and number of pulmonary complications $(32.4 \%$ vs. $29.9 \%$, $p=0.646$ ) between the COVID-19 cohort and the control cohort. Overall, postoperative morbidity and mortality rates were comparable between both cohorts. History taking and reverse transcription polymerase chain reaction (RT-PCR) were used as preoperative screening methods to detect a possible severe acute respiratory syndrome coronavirus 2 (SARS-CoV-2) infection in all centers. No patients were diagnosed with COVID-19 pre- or postoperatively.

Conclusion. Esophageal cancer surgery during the first wave of the COVID-19 pandemic was not associated with an increase in pulmonary complications as no patients were diagnosed with COVID-19. Esophageal cancer surgery can be performed safely with the use of adequate preoperative SARS-CoV-2 screening methods.

Esophageal cancer is the sixth leading cause of cancerrelated deaths worldwide. ${ }^{1,2}$ Curative treatment for locally advanced esophageal cancer consists of esophagectomy combined with perioperative (radio-)chemotherapy., Esophagectomy is a complex surgical procedure and is 
associated with substantial morbidity, in particular postoperative pneumonia and consecutive respiratory failure. ${ }^{5-8}$

Many hospitals postponed elective surgical care during the first wave of the coronavirus disease 2019 (COVID-19) pandemic. This was necessary as medical resources were shifted to increase intensive care unit capacities, to prevent patients acquiring in-hospital severe acute respiratory syndrome coronavirus 2 (SARS-CoV-2) infections, and concerns regarding the safety of healthcare workers and patients. ${ }^{9,10}$ This strategy is supported by a recent study demonstrating that patients with a SARS-CoV-2 infection undergoing surgery have increased risk for postoperative pulmonary complications and mortality. ${ }^{11}$ Additionally, patients scheduled for esophageal cancer surgery are at high risk for symptomatic COVID-19 because of epidemiologic characteristics such as high age, male sex, and high prevalence of obesity; immunosuppression due to neoadjuvant therapy; high prevalence of pre-existing pulmonary comorbidities; and transthoracic esophagectomy with single lung ventilation. ${ }^{12-14}$

On the other hand, some countries have implemented national guidelines advising the use of preoperative SARSCoV-2 screening methods in order to continue elective surgery. ${ }^{15}$ Certain international tertiary hospitals specializing in esophageal cancer have been able to continue elective cancer surgery with the use of preoperative screening; however, there is no evidence supporting the safety of this strategy as postoperative outcomes after esophageal cancer surgery during the COVID-19 pandemic have not yet been investigated in detail.

Currently, second waves of COVID-19 are occurring around the world; therefore, it is important to investigate the safety of continuing elective cancer surgery as postponement substantially increases the number of avoidable cancer deaths, as demonstrated in a recent national cancer registry analysis. ${ }^{16}$ The aim of this current study was to assess the safety of patients undergoing elective esophageal cancer surgery during the COVID-19 pandemic, focusing on respiratory failure as the most critical condition of SARS-CoV-2 infection.

\section{METHODS}

This international, retrospective, multicenter cohort study was conducted at four European tertiary referral hospitals in The Netherlands, Germany, Belgium, and Sweden, all specializing in esophageal cancer surgery. All participating centers continued elective esophageal cancer surgery during the first wave of the COVID-19 pandemic. Ethical approval was waived by the Amsterdam UMC review board because of the observational nature of the study; this decision was approved by the Institutional Review Board of each participating center.

\section{Study Population}

Consecutive adult patients undergoing elective esophageal cancer surgery were included in two cohorts. The first cohort consisted of patients who underwent esophagectomy between 1 October 2019 and 29 February 2020, i.e. the control cohort, while the second cohort consisted of patients operated between 1 March 2020 and 31 May 2020, i.e. the COVID-19 pandemic cohort. This study period reflects the months with the highest COVID-19 prevalence in the participating countries (Fig. 1).

Patients with the following characteristics were eligible for inclusion: age $\geq 18$ years and undergoing either a thoracophrenicolaparotomy, transthoracic, or transhiatal esophagectomy, which could be performed as an open or totally minimally invasive procedure (including a hybrid procedure). Pre- and postoperative testing information for SARS-CoV-2 had to be available for patients in the COVID-19 pandemic cohort. Patients undergoing emergency esophagectomy were excluded. Patient data were only used if patients did not opt out for participation in this study.

\section{Severe Acute Respiratory Syndrome Coronavirus 2 (SARS-CoV-2) Testing}

Each participating center provided information on the type of pre- and postoperative screening methods used in the COVID-19 cohort. In the case of a positive preoperative reverse transcription polymerase chain reaction (RTPCR), surgery would be postponed for 2 weeks. A repeat RT-PCR test would be performed 2 days before the new date of surgery, although repeated PCR testing is known to be of limited value and is not advised in all national multidisciplinary guidelines. ${ }^{17}$ However, in the first wave, knowledge on COVID-19 was limited and repeat RT-PCR testing was performed because of the fear of in-hospital transmission.

\section{Data Collection}

Patient demographics (age, sex, American Society of Anesthesiologists [ASA] score, and Charlson Comorbidity Index), tumor and treatment characteristics (histopathological staging, neoadjuvant therapy, type of surgery performed), and postoperative outcomes according to the Esophageal Complications Consensus Group (ECCG) definitions ${ }^{18}$ were collected from prospectively maintained databases in all centers. Additionally, pre- and 
FIG. 1 Daily number of new proven COVID-19 cases for the country of each participating center, calculated as the cumulative number for 14 days of COVID-19 cases per 100,000 population. ${ }^{33}$ COVID-19 coronavirus disease 2019

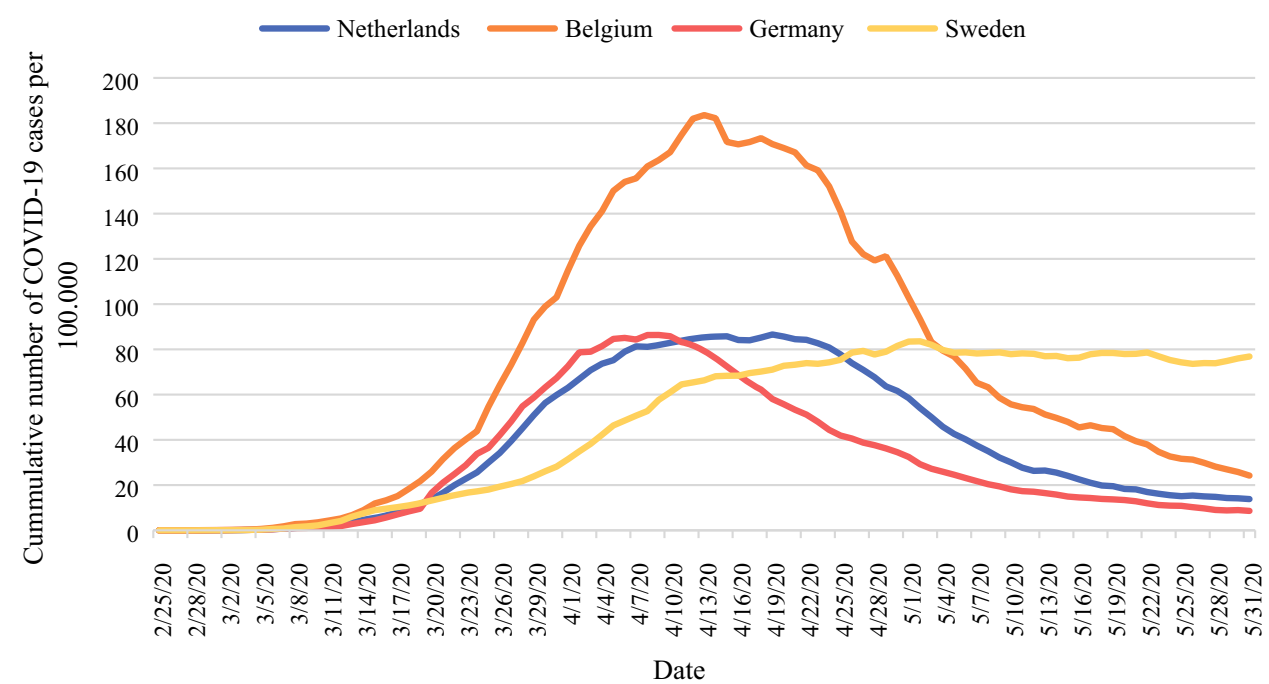

\section{RESULTS}

\section{Patient Characteristics}

Between 1 March 2020 and 31 May 2020, a total of 139 patients underwent esophageal resection for cancer in the COVID-19 pandemic cohort. A total of 168 patients were included in the control cohort between 1 October 2019 and 29 February 2020. Baseline and treatment characteristics of all patients in both cohorts are presented in Table 1 . Patients operated during the COVID-19 pandemic had a significantly higher ASA score. There were no differences in tumor and treatment characteristics between patients in both cohorts. Almost $75 \%$ of all patients in both cohorts underwent a minimally invasive esophagectomy.

\section{Postoperative Outcomes}

Table 2 shows the postoperative outcomes of all patients in both cohorts. There was no difference in the total number of postoperative complications $(64.0 \%$ vs. $63.7 \%$, $p=0.951)$, mean CCI score (44.3 vs. 39.7, $p=0.699)$, and maximum Clavien-Dindo score $(p=0.317)$.

The percentage of respiratory failures requiring mechanical ventilation $(13.7 \%$ vs. $8.3 \%, p=0.127)$ and the total number of pulmonary complications were comparable between both cohorts $(32.4 \%$ vs. $29.9 \%$, $p=0.647)$. The ICU admission rate and length of stay at the ICU were similar in both cohorts. In one center, all patients were admitted to the ICU postoperatively as part of standard care (electronic supplementary Table S1). There was no difference in the 30-day readmission and mortality rates between the COVID-19 pandemic and control cohorts. 
TABLE 1 Baseline and treatment characteristics of all patients undergoing esophageal cancer surgery, as compared between the COVID-19 pandemic cohort (1 March 2020-31 May 2020) and the control cohort (1 October 2020-29 February 2020)

\begin{tabular}{|c|c|c|c|}
\hline Characteristics & COVID-19 pandemic group [ $N=139]$ & Control cohort $[N=168]$ & $p$ value \\
\hline Age, years [median (IQR)] & $66(58-71)$ & $67(60-73)$ & 0.165 \\
\hline Male sex & $116(83.5)$ & $141(83.9)$ & 0.911 \\
\hline $\mathrm{BMI}>25$ & $49.3(68)$ & $100(59.5)$ & 0.073 \\
\hline ASA score & & & 0.015 \\
\hline 1 & $14(10.1)$ & $37(22.0)$ & \\
\hline 2 & $81(58.3)$ & $90(53.6)$ & \\
\hline 3 & $44(31.7)$ & $39(23.2)$ & \\
\hline 4 & $0(0.0)$ & $2(1.2)$ & \\
\hline WHO performance status & & & 0.431 \\
\hline 0 & $75(54.0)$ & $86(51.2)$ & \\
\hline 1 & $48(34.5)$ & $62(36.9)$ & \\
\hline 2 & $12(8.6)$ & $15(8.9)$ & \\
\hline 3 & $2(1.4)$ & $0(0.0)$ & \\
\hline 4 & $1(0.7)$ & $0(0.0)$ & \\
\hline Missing & $1(0.7)$ & $5(3.0)$ & \\
\hline Charlson comorbidity index & & & 0.403 \\
\hline 0 & $9(6.5)$ & $5(3.0)$ & \\
\hline 1 & $24(17.3)$ & $26(15.5)$ & \\
\hline 2 & $31(22.3)$ & $49(29.2)$ & \\
\hline 3 & $36(25.9)$ & $29(17.3)$ & \\
\hline $4+$ & $39(28.0)$ & $59(35.0)$ & \\
\hline Comorbidities & & & 0.254 \\
\hline Myocardial infarction & $6(4.3)$ & $7(4.2)$ & \\
\hline Congestive heart failure & $0(0.0)$ & $0(00)$ & \\
\hline Chronic pulmonary disease & $14(10.1)$ & $14(8.3)$ & \\
\hline Diabetes mellitus (uncomplicated) & $21(15.1)$ & $16(9.5)$ & \\
\hline Moderate to severe renal disease & $0(0.0)$ & $2(1.2)$ & \\
\hline Multiple & $4(2.9)$ & $12(7.1)$ & \\
\hline Histology & & & 0.672 \\
\hline Adenocarcinoma & $108(77.7)$ & $135(80.4)$ & \\
\hline Squamous cell carcinoma & $28(20.1)$ & $30(17.9)$ & \\
\hline Other & $3(2.2)$ & $2(1.2)$ & \\
\hline Clinical $\mathrm{T}$ stage & & & 0.416 \\
\hline cT1 & $7(5.0)$ & $8(4.8)$ & \\
\hline $\mathrm{cT} 2$ & $13(9.4)$ & $23(13.7)$ & \\
\hline cT3 & $106(76.3)$ & $124(73.8)$ & \\
\hline cT4 & $3(2.2)$ & $10(6.0)$ & \\
\hline $\mathrm{cTx}$ & $6(4.3)$ & $10(6.0)$ & \\
\hline Missing & $4(2.9)$ & $0(0.0)$ & \\
\hline Clinical N stage & & & 0.887 \\
\hline $\mathrm{cNO}$ & $33(23.7)$ & $44(26.2)$ & \\
\hline $\mathrm{cN} 1$ & $30(21.6)$ & 33 (19.6) & \\
\hline $\mathrm{cN} 2$ & 18 (12.9) & $27(16.1)$ & \\
\hline $\mathrm{cN} 3$ & $3(2.3)$ & $4(2.4)$ & \\
\hline $\mathrm{cNx}$ & $55(39.6)$ & $60(35.7)$ & \\
\hline Neoadjuvant therapy & & & 0.958 \\
\hline Chemotherapy & $36(25.9)$ & $46(27.4)$ & \\
\hline Chemoradiotherapy & 77 (55.4) & $91(54.2)$ & \\
\hline
\end{tabular}


TABLE 1 (continued)

\begin{tabular}{lcc}
\hline Characteristics & COVID-19 pandemic group $[N=139]$ & Control cohort $[N=168]$ \\
\hline Surgical approach & & $p$ value \\
Open & $35(25.2)$ & $40(23.8)$ \\
Minimally invasive & $104(74.8)$ & $125(74.4)$ \\
Minimally invasive converted to open & $0(0.0)$ & $3(1.8)$ \\
Esophagectomy & & $4(2.4)$ \\
Transhiatal & $3(2.2)$ & $138(82.1)$ \\
Transthoracic & $119(85.6)$ & $26(15.5)$ \\
Thoracophrenicolaparotomy & $17(12.2)$ & 0.705 \\
\hline
\end{tabular}

Data are expressed as $n(\%)$ unless otherwise specified

ASA American Society of Anesthesiologists, BMI body mass index, COVID-19 coronavirus disease 2019, IQR interquartile range

TABLE 2 Postoperative outcomes of all patients undergoing esophageal cancer surgery, as compared between the COVID-19 pandemic cohort and the control cohort

\begin{tabular}{|c|c|c|c|}
\hline & COVID-19 pandemic group [ $N=139]$ & Control cohort $[N=168]$ & $p$ value \\
\hline \multicolumn{4}{|l|}{ Complications } \\
\hline Yes & $89(64.0)$ & $107(63.7)$ & 0.951 \\
\hline CCI score [mean (SD)] & $41.2(25.5)$ & $39.8(20.2)$ & 0.699 \\
\hline Maximum Clavien-Dindo & & & 0.317 \\
\hline I & $5(3.6)$ & $8(4.8)$ & \\
\hline II & $33(23.7)$ & $35(20.8)$ & \\
\hline III & $25(18.0)$ & $39(23.2)$ & \\
\hline IV & $25(18.0)$ & $24(14.3)$ & \\
\hline V & $5(3.6)$ & $2(1.2)$ & \\
\hline Pulmonary complications & $45(32.4)$ & $50(29.9)$ & 0.647 \\
\hline Pneumonia & $20(14.4)$ & $32(19.0)$ & 0.297 \\
\hline Respiratory failure requiring reintubation & $19(13.7)$ & $14(8.3)$ & 0.127 \\
\hline ICU admission & 69 (49.6) & $98(58.3)$ & 0.128 \\
\hline ICU admission, days [median (IQR)] & $0(0-4)$ & $1(0-3)$ & 0.686 \\
\hline Length of hospital stay, days [median (IQR)] & $12(9-16.25)$ & $12.5(9-17.75)$ & 0.430 \\
\hline \multicolumn{4}{|l|}{ Readmission within 30 days } \\
\hline Yes & $16(11.5)$ & $14(8.3)$ & 0.184 \\
\hline \multicolumn{4}{|l|}{ 30-day mortality } \\
\hline Yes & $5(3.6)$ & $3(1.8)$ & 0.263 \\
\hline
\end{tabular}

Data are expressed as $n(\%)$ unless otherwise specified

CCI Comprehensive Complications Index, COVID-19 coronavirus disease 2019, ICU intensive care unit, IQR interquartile range, $S D$ standard deviation

Electronic supplementary Table S1 provides an overview of the postoperative outcomes for each of the participating centers; no statistical differences between centers were observed.

In univariate logistic regression analysis, the odds ratio (OR) for postoperative respiratory failure requiring mechanical ventilation was 1.44 (95\% confidence interval [CI] $0.80-2.58, p=0.222$ ) for patients operated during the
COVID-19 pandemic. In a multivariate logistic regression, adjusted for ASA score, surgical approach and surgical procedure, the OR was $1.43(95 \%$ CI $0.76-2.70, p=0.272$ ) for patients in the COVID-19 pandemic group. 


\section{SARS-CoV-2 Testing Results}

An overview of the screening methods used in each center is provided in electronic supplementary Table S2. All centers used COVID-19-specific symptom screening and RT-PCR; however, the date of implementation of the screening methods was different in each hospital.

SARS-CoV-2 testing results of patients in the COVID19 pandemic cohort are presented in Table 3. Overall, 134/139 (96.4\%) patients were screened for COVID-19 preoperatively and all were negative. History taking for specific COVID-19 symptoms was performed in most patients $(95.0 \%)$, followed by white cell/lymphocyte count (73.4\%), RT-PCR (71.9\%), and chest computed tomography (CT; $11.5 \%)$. Thirty-six symptomatic patients $(25.9 \%)$ received postoperative RT-PCR testing for SARS-CoV-2 and all patients tested negative. Overall, none of the patients were diagnosed with COVID-19, and subsequently no surgery was postponed because of screening results.

\section{DISCUSSION}

This study investigated the safety of patients undergoing elective esophageal cancer surgery during the first wave of the COVID-19 pandemic in Europe and compared that with patients undergoing surgery in a period just before the COVID-19 pandemic. None of the patients in the COVID19 pandemic cohort were pre- or postoperatively diagnosed with COVID-19. This resulted in a similar rate of patients with respiratory failure requiring mechanical ventilation in both cohorts. Therefore, undergoing esophagectomy during the COVID-19 pandemic was not associated with an increased risk of respiratory failure.
The ICU admission rate and length of stay at the ICU were comparable between both cohorts. In one center, all patients went to the ICU postoperatively as part of standard care. None of the participating centers experienced a shortage of ICU beds or delay in ICU readmission because of hospital COVID-19 volume during our inclusion period. The ASA score was higher in the COVID-19 pandemic cohort, with a higher percentage of patients with an ASA score of 2-3. There was no specific reason for this difference.

This is the first study to investigate the short-term postoperative outcomes in patients undergoing elective esophageal cancer surgery during the COVID-19 pandemic.

In the COVID-19 cohort, the percentage of patients with pulmonary complications (32.4\%), the rate of respiratory failure requiring mechanical ventilation $(13.7 \%)$, and the 30-day mortality rate $(3.6 \%)$ were comparable with the findings of previous studies. ${ }^{7,8,21}$

A recent study by Chenchen et al. investigated the safety of performing cancer surgery during the COVID-19 pandemic. $^{22}$ They found that none of the 621 patients tested positive for COVID-19 postoperatively. Shrikhande et al. performed a single-center prospective study examining 494 patients undergoing elective major cancer surgery in India and found that only six patients were diagnosed with COVID-19 postoperatively, none of whom required escalating care or intensive care treatment. ${ }^{23}$ In line with these findings, no patients were diagnosed with COVID-19 in our cohort, although the COVID-19 community prevalence in Europe was higher in our study period.

Studies performed at the beginning of the COVID-19 pandemic concluded that patients with a SARS-CoV-2 infection undergoing surgery had worse postoperative
TABLE 3 Pre- and postoperative SARS-CoV-2 testing results of patients in the COVID-19 pandemic cohort

\begin{tabular}{lccc}
\hline Preoperative & $N=139$ & Postoperative & $N=139$ \\
\hline COVID-19 & $0(0.0)$ & COVID-19 & \\
Positive & $134(96.4)$ & Positive & Negative \\
Negative & $5(3.6)$ & Not tested & $36(25.9)$ \\
Not tested & & Methods & $103(74.1)$ \\
Methods & $100(71.9)$ & RT-PCR & $36(25.9)$ \\
RT-PCR & $16(11.5)$ & & \\
Chest CT & $132(95.0)$ & & \\
Symptom screening & $102(73.4)$ & & \\
White-cell/lymphocyte count & $0(0.0)$ & & \\
Antibody analysis & $139(100.0)$ & & \\
Surgery postponed & & \\
No & & & \\
\hline
\end{tabular}

Data are expressed as $n(\%)$

COVID-19 coronavirus disease 2019, RT-PCR reverse transcription polymerase chain reaction, $C T$ computed tomography, $S A R S-\mathrm{CoV}-2$ severe acute respiratory syndrome coronavirus 2 
outcomes, with a high postoperative mortality rate. $^{24}$ Increased 30-day mortality was associated with male sex, age $>70$ years, ASA score of 3-5, cancer surgery, and major surgical procedures. Additionally, oncologic patients undergoing surgery or chemotherapy have increased risk for severe COVID-19. ${ }^{14}$ Based on these findings, international societies advised to postpone elective surgery when possible, including esophageal cancer surgery. ${ }^{13} \mathrm{~A}$ study by the COVIDSurg group has estimated that the total percentage of adult elective operations that were cancelled during the first 12 weeks of the first wave of the COVID-19 pandemic was $72.3 \% .^{25}$ Globally, $37.7 \%$ of all cancer operations were cancelled or postponed. The study concluded that if countries increased their normal surgical capacity by $20 \%$ after the COVID-19 pandemic, it would take a median of 45 weeks to clear the accumulation of operations. ${ }^{25}$ The question remains whether postponement of cancer surgery leads to progression of the tumor and reduced overall survival. Turaga and Girotra investigated how long different types of cancer surgery could be safely delayed and concluded that most cancer surgeries can be safely delayed for at least 4 weeks without having a significant impact on patient survival or cancer progression. ${ }^{26}$ However, with a second COVID-19 wave currently developing in Europe, waiting lists will start to increase, which might lead to postponement of elective cancer surgery for more than 4 weeks.

A Dutch study evaluated the yield of preoperative screening for COVID-19 with chest CT and RT-PCR in asymptomatic patients. RT-PCR detected SARS-CoV-2 in at least 1 in every 100 asymptomatic patients undergoing elective or emergency surgery. ${ }^{27}$ This yield increased to $6 \%$ when the COVID-19 daily hospital admissions rate exceeded 1.5 per 100,000 inhabitants. The incremental yield of chest CT was only $0.4 \%$ and did not contribute to COVID-19 detection. None of the patients who underwent history taking and RT-PCR preoperatively developed symptomatic COVID-19 after surgery. ${ }^{27}$ In line with these findings, a recent study by the COVIDSurg group concluded that preoperative RT-PCR testing was beneficial before major surgery and in high SARS-CoV-2 risk areas. ${ }^{28}$ Having at least one negative preoperative RT-PCR test was associated with a lower rate of pulmonary complications (OR 0.68, 95 CI 0.68-0.98, $p=0.040){ }^{28}$

In our study, almost all patients in the COVID-19 cohort were screened for COVID-19-specific symptoms preoperatively and $70 \%$ underwent RT-PCR testing. RT-PCR testing was used as the standard preoperative screening method in all participating centers; however, because of limited testing capacity and differences in implications of national guidelines at the beginning of the COVID-19 pandemic, not all patients were tested with RT-PCR. Only symptomatic patients underwent RT-PCR testing for COVID-19 postoperatively.

Surgery would have been postponed for 2 weeks if a patient tested positive for COVID-19 preoperatively, with an additional RT-PCR test 2 days before the new date of surgery. This strategy would have been applied irrespective of whether a patient received neoadjuvant therapy. A previous study found that the interval between neoadjuvant therapy and esophagectomy could be safely extended to a maximum of 10 or more weeks. ${ }^{29}$

Seventy-five percent of all patients underwent minimally invasive surgery in both the COVID-19 pandemic cohort and the control cohort. According to the Society of American Gastrointestinal and Endoscopic Surgeons (SAGES) and the European Association of Endoscopic Surgery (EAES) guidelines, released during the first wave of the COVID-19 pandemic, minimally invasive surgery was discouraged as these procedures could contaminate surgical staff. ${ }^{30}$ Our study did not investigate the rate of SARS-CoV-2 infections among the surgical staff; however, as all patients were screened for COVID-19 preoperatively, the strategy of performing minimally invasive surgery in our cohort was safe.

Our study has some limitations. First, patients were included retrospectively from prospectively maintained databases in all centers; therefore, the participating centers did not use a similar standardized preoperative COVID-19 screening strategy during the inclusion period. Second, not all patients in the COVID-19 cohort were screened for COVID-19 preoperatively and $75 \%$ of patients were not tested postoperatively. Furthermore, only RT-PCR was used postoperatively to diagnose possible COVID-19 cases. RT-PCR is considered the reference standard to establish a SARS-CoV-2 infection, however sensitivity is considered to be moderate. ${ }^{31}$ Hence, asymptomatic COVID-19 patients or patients with false negative testing results could have been missed. However, clinical followup information was obtained for all patients and no patients were suspected for symptomatic COVID-19. Third, surgeons may have selected the healthiest patients to undergo surgery during the peak of the COVID-19 pandemic; therefore, this patient group might not be representative of the normal population undergoing esophageal cancer surgery. However, no differences were found in baseline characteristics between both cohorts.

We are currently facing a second COVID-19 wave in Europe, which is characterized by the appearance of new SARS-CoV-2 variants. ${ }^{31}$ Although it is unknown whether these new variants are more infectious, epidemiological data show that these variants have a higher transmissibility compared with the original variant. ${ }^{31,32}$ Hospitals will therefore face a higher number of COVID-19 patients 
during the upcoming months, which may affect the surgical and ICU capacity. Hence, hospitals that could continue elective cancer surgery during the first COVID-19 wave might have problems continuing cancer care during the second and possibly third waves. Increased lockdown measures and vaccination might prevent such a scenario.

\section{CONCLUSION}

Elective esophageal cancer surgery can be performed safely during the COVID-19 pandemic with the use of adequate preoperative SARS-CoV-2 screening methods. With increasing numbers of operations being cancelled or postponed around the world, this study indicates that patients can undergo major cancer surgery during the ongoing COVID-19 pandemic without additional risk for the patient.

FUNDING No external funding was received for this study.

DISCLOSURE Mark I. van Berge Henegouwen is a consultant for Mylan, Alesi Surgical, Medtronic, and Johnson and Johnson, and has received research grants from Olympus and Stryker, paid to the institute. Hans Fuchs is on the advisory board of Activ Surgical, received an educational grant from Intuitive Surgical and Stryker, and holds stock options for Fortimedix Surgical. Christiane J. Bruns has receive support for presentations from Celgene, MCI Health Care Academy, and Update Chirurgie; support for conferences and local hospitations from Medtronic and Intuitive; and research support from SIRTex, Intuitive, and Servier for clinical trials. Alexander B.J. Borgstein, Stefanie Brunner, Masaru Hayami, Johnny Moons, Wietse J. Eshuis, Suzanne S. Gisbertz, Philippe Nafteux, Magnus Nilsson, and Wolfgang Schröder declare no competing interests.

OPEN ACCESS This article is licensed under a Creative Commons Attribution 4.0 International License, which permits use, sharing, adaptation, distribution and reproduction in any medium or format, as long as you give appropriate credit to the original author(s) and the source, provide a link to the Creative Commons licence, and indicate if changes were made. The images or other third party material in this article are included in the article's Creative Commons licence, unless indicated otherwise in a credit line to the material. If material is not included in the article's Creative Commons licence and your intended use is not permitted by statutory regulation or exceeds the permitted use, you will need to obtain permission directly from the copyright holder. To view a copy of this licence, visit http://creativecommons. org/licenses/by/4.0/.

\section{REFERENCES}

1. Ferlay J, Shin HR, Bray F, Forman D, Mathers C, Parkin DM. Estimates of worldwide burden of cancer in 2008: GLOBOCAN 2008. Int J Cancer. 2010;127(12):2893-917. https://doi.org/10. 1002/ijc. 25516.

2. Arnold M, Ferlay J, Van Berge Henegouwen MI, Soerjomataram I. Global burden of oesophageal and gastric cancer by histology and subsite in 2018. Gut. 2020;69(9):1564-71. https://doi.org/10. 1136/gutjnl-2020-321600.
3. Lordick F, Mariette C, Haustermans K, Obermannová R, Arnold $\mathrm{D}$, on behalf of the ESMO Guidelines Committee. Oesophageal cancer: ESMO clinical practice guidelines for diagnosis, treatment and follow-up. Ann Oncol. 2016;27:v50-7. https://doi.org/ 10.1093/annonc/mdw329.

4. Kitagawa Y, Uno T, Oyama T, et al. Esophageal cancer practice guidelines 2017 edited by the Japan esophageal society: part 2 . Esophagus. 2019;16(1):25-43. https://doi.org/10.1007/s10388-0 18-0642-8.

5. Low DE, Kuppusamy MK, Alderson D, et al. Benchmarking complications associated with esophagectomy. Ann Surg. 2019;269(2):291-8. https://doi.org/10.1097/SLA. 0000000000002611.

6. Van Der Werf LR, Busweiler LAD, Van Sandick JW, Van Berge Henegouwen MI, Wijnhoven BPL. Reporting national outcomes after esophagectomy and gastrectomy according to the Esophageal Complications Consensus Group (ECCG). Ann Surg. 2020;271(6):1095-101. https://doi.org/10.1097/SLA. 0000000000003210.

7. Schröder W, Raptis DA, Schmidt HM, et al. Anastomotic techniques and associated morbidity in total minimally invasive transthoracic esophagectomy: results from the EsoBenchmark Database. Ann Surg. 2019;270(5):820-6. https://doi.org/10.1097/ SLA.0000000000003538.

8. Kuppusamy MK, Low DE. Evaluation of international contemporary operative outcomes and management trends associated with esophagectomy: a 4-year study of $>6000$ patients using ECCG definitions and the online Esodata Database. Ann Surg. 2020. https://doi.org/10.1097/sla.0000000000004309.

9. Besnier E, Tuech JJ, Schwarz L. We asked the experts: Covid-19 outbreak: is there still a place for scheduled surgery? "Reflection from pathophysiological data." World J Surg. 2020;44(6):1695-8. https://doi.org/10.1007/s00268-020-05501-6.

10. Aminian A, Safari S, Razeghian-Jahromi A, Ghorbani M, Delaney CP. COVID-19 outbreak and surgical practice: unexpected fatality in perioperative period. Ann Surg. 2020;272(1):e27-9. h ttps://doi.org/10.1097/SLA.0000000000003925.

11. Nepogodiev D, Bhangu A, Glasbey JC, et al. Mortality and pulmonary complications in patients undergoing surgery with perioperative SARS-CoV-2 infection: an international cohort study. Lancet. 2020;396(10243):27-38. https://doi.org/10.1016/ S0140-6736(20)31182-X.

12. McLoughlin JM, Lewis JM, Meredith KL. The impact of age on morbidity and mortality following esophagectomy for esophageal cancer. Cancer Control. 2013;20(2):144-50. https://doi.org/10.1 177/107327481302000208.

13. Barbieri L, Urquijo ET, Parise P, Nilsson M, Reynolds JV, Rosati R. Esophageal oncologic surgery in SARS-CoV-2 (COVID-19) emergency. Dis Esophagus. 2020;33(5):1-5. https://doi.org/10.1 093/dote/doaa028.

14. Liang W, Guan W, Chen R, et al. Cancer patients in SARS-CoV2 infection: a nationwide analysis in China. Lancet Oncol. 2020;21(3):335-7. https://doi.org/10.1016/S1470-2045(20)30096 $-6$.

15. This D, Sars-cov-IT, Specialists M. Practice guideline Leidraad. 2020. p. 1-11.

16. Bertuzzi AF, Marrari A, Gennaro N, et al. Low incidence of SARS-CoV-2 in patients with solid tumours on active treatment: an observational study at a Tertiary Cancer Centre in Lombardy, Italy. Cancers (Basel). 2020;12(9):2352. https://doi.org/10.3390/ cancers12092352.

17. Nederlandse Vereniging voor Heelkunde (NVVH). Pre-operatieve diagnostiek naar SARS-CoV-2 infectie bij asymptomatische patiënten ingepland voor chirurgie onder algehele anesthesie. 2020. p. 1-18. 
18. Low DE, Alderson D, Cecconello I, et al. International consensus on standardization of data collection for complications associated with esophagectomy: Esophagectomy Complications Consensus Group (ECCG). Ann Surg. 2015;262(2):286-94. https://doi.org/ 10.1097/SLA.0000000000001098.

19. Dindo D, Demartines N, Clavien PA. Classification of surgical complications: a new proposal with evaluation in a cohort of 6336 patients and results of a survey. Ann Surg. 2004;240(2):205-13. https://doi.org/10.1097/01.sla.0000133083. 54934.ae.

20. Slankamenac K, Graf R, Barkun J, Puhan MA, Clavien PA. The comprehensive complication index: a novel continuous scale to measure surgical morbidity. Ann Surg. 2013;258(1):1-7. https://d oi.org/10.1097/SLA.0b013e318296c732.

21. Merkow RP, Bilimoria KY, McCarter MD, et al. Short-term outcomes after esophagectomy at 164 American College of Surgeons National Surgical Quality Improvement Program Hospitals: effect of operative approach and hospital-level variation. Arch Surg. 2012;147(11):1009-16. https://doi.org/10.1001/ 2013.jamasurg.96.

22. Chenchen J, Singh K, Luther AZ, Agrawal A. Is elective cancer surgery safe during the COVID-19 pandemic? World J Surg. 2020;44(10):3207-11. https://doi.org/10.1007/s00268-020-05720 $-\mathrm{x}$

23. Shrikhande SV, Pai PS, Bhandare MS, et al. Outcomes of elective major cancer surgery during COVID 19 at Tata Memorial Centre. Ann Surg. 2020;272(3):e249-52. https://doi.org/10.1097/sla. 0000000000004116

24. Nepogodiev D, Glasbey JC, Li E, et al. Mortality and pulmonary complications in patients undergoing surgery with perioperative SARS-CoV-2 infection: an international cohort study. Lancet. 2020;396(10243):27-38. https://doi.org/10.1016/S0140-6736(20) 31182-X.

25. COVIDSurg Collaborative. Elective surgery cancellations due to the COVID-19 pandemic: global predictive modelling to inform surgical recovery plans. Br J Surg. 2020. https://doi.org/10.100 2/bjs.11746.

26. Turaga KK, Girotra S. Are we harming cancer patients by delaying their cancer surgery during the COVID-19 pandemic?
Ann Surg. 2020. https://doi.org/10.1097/SLA. 0000000000003967.

27. Puylaert CAJ, Scheijmans JCG, Borgstein ABJ, et al. Yield of screening for COVID-19 in asymptomatic patients prior to elective or emergency surgery using chest CT and RT-PCR (SCOUT). Ann Surg. 2020;272(6):919-24. https://doi.org/10.10 97/sla.0000000000004218.

28. COVIDSurg Collaborative. Preoperative nasopharyngeal swab testing and postoperative pulmonary complications in patients undergoing elective surgery during the SARS-CoV-2 pandemic. Br J Surg. 2021;108(1):88-96. https://doi.org/10.1093/bjs/znaa 051.

29. van der Werf LR, Dikken JL, van der Willik EM, et al. Time interval between neoadjuvant chemoradiotherapy and surgery for oesophageal or junctional cancer: a nationwide study. Eur $J$ Cancer. 2018;91:76-85. https://doi.org/10.1016/j.ejca.2017.12. 009.

30. SAGES and EAES Recommendations Regarding Surgical Response to COVID-19 Crisis. Available at: https://www.sages. org/recommendations-surgical-response-covid-19/. Accessed 20 Nov 2020.

31. Fang Y, Zhang H, Xie J, et al. Sensitivity of chest CT for COVID-19: comparison to RT-PCR. Radiology. 2020;296(2):E115-7. https://doi.org/10.1148/radiol.2020200432.

32. World Health Ogranization. SARS-CoV-2 Variant - United Kingdom of Great Britain and Northern Ireland. Available at: $h$ ttps://www.who.int/csr/don/21-december-2020-sars-cov2-varian t-united-kingdom/en/. Accessed 28 Jan 2021.

33. Download the daily number of new reported cases of COVID-19 by country worldwide. Available at: https://www.ecdc.europa.e u/en/publications-data/download-todays-data-geographic-distribu tion-covid-19-cases-worldwide. Accessed 16 Oct 2020.

Publisher's Note Springer Nature remains neutral with regard to jurisdictional claims in published maps and institutional affiliations. 